\title{
Hybrid Vortex/Magnet Methods for Flow Over a Solid Boundary*
}

\author{
David M. Summers \\ Mathematics Department \\ Napier University \\ 219 Colinton Road \\ Edinburgh, EH14 1DJ, Scotland
}

\author{
Alexandre J. Chorin \\ Department of Mathematics \\ University of California \\ Berkeley, CA94720, USA
}

\begin{abstract}
Boundary conditions, in particular no-slip boundary conditions, are usually imposed in vortex methods through the creation of vorticity. In three dimensions, this is typically done by creating vortex "blobs" or "segments". However, with these computational elements, one compromises accuracy by losing the divergence-free nature of the vorticity field. Furthermore, these methods preclude the use of hairpin removal strategies for simplifying the calculation. We explore remedies for this problem through the use of discrete elements of fluid impulse (also known as "magnets"). In particular, two strategies for evolving impulse from a boundary, consistent with the no-slip condition, are proposed; they correspond to two choices of gauge. Sheet-like elements are created at walls to carry this impulse; as these elements diffuse away from the wall into the flow interior they are transformed into vortex loops of equal impulse. In this way hybrid vortex algorithms are determined for three-dimensional incompressible bounded flow. These ideas are illustrated by numerical experiment with high Reynolds number flow past a sphere.
\end{abstract}

\section{Introduction}

Vortex methods in three-dimensions typically enforce no-slip boundary conditions by the creation of disjoint three-dimensional vortex blobs, arrows, or segments, which subsequently evolve according to their transport equation. Typically the divergence-free property of the vorticity field is not enforced exactly during the evolution, and this can lead to loss of accuracy, manifesting itself, for example, in rapidly growing instability in the time-evolution. Furthermore, the use of non-solenoidal elements precludes the use of hairpin removal and renormalization strategies [6].

On the other hand, Buttke's [3] reformulation of vortex methods in terms of impulse elements naturally preserves the divergence-free character of vorticity. In order to use this formalism in a problem of viscous flow bounded by solid walls, one needs a boundary condition for impulse which enforces no-slip at the solid boundary. We consider the problem of bounded exterior flow; namely flow in $\mathcal{B} \in \mathbb{R}^{3}$ with an interior boundary surface $\partial \mathcal{B}$. The specific example we will consider is flow over a sphere.

The impulse (or magnetization) $\mathbf{m}$ is defined in terms of vorticity $\boldsymbol{\xi}$ as $\boldsymbol{\xi}=\nabla \times \mathbf{m}$. The identity $\nabla \times \mathbf{m} \equiv \nabla \times \mathbf{u}$ implies that velocity $\mathbf{u}$ and impulse $\mathbf{m}$ are equal up to an additive gradient,

$$
\mathbf{m}=\mathbf{u}+\nabla \phi
$$

${ }^{*}$ This work was supported in part by the Applied Mathematical Sciences Subprogram of the Office of Energy Research, U.S. Department of Energy under Contract DE-AC03-76SF00098. 
where $\phi$ is a scalar function. This is a gauge freedom which implies that neither $\mathbf{m}$ nor $\phi$ is uniquely determined. This freedom makes it possible to localize $\mathbf{m}$, i.e., to choose $\phi$ so that the support of $\mathbf{m}$ is bounded if the support of the vorticity is bounded [3].

One natural generalization of familiar vortex methods (with vorticity created tangential to $\partial \mathcal{B}$ ) is achieved by choosing $\mathbf{m}$ perpendicular to $\partial \mathcal{B}$. This leads to an algorithm which embodies the creation and subsequent conservation of circulation. An alternative algorithm can be based on the choice of $\mathbf{m}$ parallel to $\partial \mathcal{B}$. At a given time step this implies the creation of an impulse density at the wall equal to the 'slip-flow' there, and opposite in direction. This enforces momentum conservation.

There is a simple relationship between impulse of compact support and an equivalent vortex loop; the impulse equals the product of the circulation of the loop and its spanned area. Given an impulse element one can construct a filament loop of equal impulse with which to replace it. This matching of impulse allows a creation algorithm for impulse at a solid wall to be used to generate a system of vortex loops at an interface between a wall boundary-layer and the flow interior. Vortex loops created in this way will be consistent with a no-slip condition, and their subsequent evolution will preserve the solendoidal character of the vorticity field. Representing the flow in the interior by an ensemble of closed vortex loops of uniform circulation has the additional advantage that hairpin-removal strategies can be invoked to reduce the computational effort.

Since there exists $[3,4]$ an evolution equation for impulse in the interior, it is possible to cast the entire flow problem in terms of impulse. However the use of impulse elements in the interior poses a number of difficult numerical problems. The strength of the elements increases very rapidly as they evolve, placing serious demands on the accuracy of the time-integration. This problem is avoided if impulse elements are used only transitionally at walls.

In order to illustrate the potential usefulness of this approach to solving for bounded flow problems, we apply these ideas to high-Reynolds number flow past a sphere. We discuss how forces and impulse are to be calculated in a flow described by closed-loop filaments.

\section{Evolution of impulse in unbounded flow}

The equations of motion for incompressible viscous flow are the Navier-Stokes equations:

$$
\frac{\partial \mathbf{u}}{\partial t}+\mathbf{u} \cdot \nabla \mathbf{u}=\nu \Delta \mathbf{u}-\nabla p
$$

where $\mathbf{u}$ is the velocity satisfying the condition $\nabla \cdot \mathbf{u}=0$, and $p$ is the pressure (we assume uniform unit density, $\rho=1$ ). The parameter $\nu$ is the kinematic viscosity.

The divergence of (1) yields:

$$
\Delta \phi=\nabla \cdot \mathbf{m}
$$

Hence we can express the velocity in terms of the impulse as

$$
\mathbf{u}=\mathbf{m}-\nabla\left\{\Delta^{-1}\{\nabla \cdot \mathbf{m}\}\right\}
$$

where $\Delta^{-1}$ represents the inverse Laplace operator.

Considering the curl of the Navier-Stokes equation (2), and invoking vector identities and the equality $\nabla \times \mathbf{u}=\nabla \times \mathbf{m}$, we can verify that the evolution equation for $\mathbf{m}$,

$$
\frac{\partial \mathbf{m}}{\partial t}+\mathbf{u} \cdot \nabla \mathbf{m}+(\nabla \mathbf{u})^{T} \mathbf{m}=\nu \Delta \mathbf{m}
$$

is equivalent to (2) in the absence of walls. This is the gauge invariant form of (2). (In Eq. 5 the $i j$ element of the matrix $(\nabla \mathbf{u})^{T}$ is $\partial u_{j} / \partial x_{i}$.) 
The vector $\mathbf{u}$ in a region $\Omega$ can be interpreted as the orthogonal projection of $\mathbf{m}$ in $\Omega$ on the space of solenoidal vectors parallel to the boundary $\partial \Omega$. This is to emphasize that $\mathbf{m}$ is not generally solenoidal, although the requirement that it be solenoidal could, of course, be a particular choice of gauge. There exists a unique operator that projects $\mathbf{m}$ on the space of solenoidal velocities $\mathbf{u}$.

Total impulse, a global quantity, is defined in terms of vorticity as an integral over volume:

$$
\mathbf{M}=\frac{1}{2} \int_{\mathbb{R}^{3}}(\mathbf{r} \times \boldsymbol{\xi}) d V
$$

or, equivalently,

$$
\mathbf{M}=\frac{1}{2} \int_{\mathbb{R}^{3}}(\mathbf{r} \times \nabla \times \mathbf{m}) d V
$$

After integration by parts, this is

$$
\mathbf{M}=\int_{\mathbb{R}^{3}} \mathbf{m} d V
$$

Thus $\mathbf{m}$ can be interpreted as an impulse density.

Equation 6 shows in particular that in the case of a single loop we have $|\mathbf{M}|=\Gamma A_{\text {loop }}$ where $\Gamma$ is the circulation of the loop, and $A_{\text {loop }}$ is its spanned area (see [11]).

\section{Choice of gauge}

Vortex methods applied to bounded flow are based on the application of the Kelvin-Helmholtz circulation theorem to vortex elements. At a given time circulation is created at a wall to extinguish the 'slip-field'. This circulation is typically attached to a vortex sheet which is made to diffuse vertically from the wall in a "particle" modelling of the Prandtl boundary layer equations [5]. Upon leaving the neighbourhood of the boundary, these sheets are transformed into vortex segments (or blobs) bearing the circulation created at the wall. These segments are parallel to the wall and perpendicular to the slip-field at point of creation. The segments are then transported in the flow, to which they contribute an induced field.

This boundary layer approximation can be recast in terms of impulse variables, leading to the evolution of tangential vortex elements from the surface in the form of vortex loops. As these elements are transported they preserve the solenoidal character of the vorticity field. This class of methods leads to $\mathbf{m}$ normal to the wall.

Another choice of gauge makes $\mathbf{m}$ parallel to the wall. This can be interpreted as allowing the tangential boundary forces to impart impulse to the fluid directly. The two choices lead to the same vorticity field in a fluid continuum, but they are not necessarily numerically equivalent; the choice of gauge determines where the impulse elements and the vortex elements that they eventually produce are concentrated; the best choice of gauge is likely to depend of what part of the flow one is most interested in. The analysis of the best choice remains to be done.

\section{Impulse and drag}

Impulse is created to enforce no-slip at a solid boundary $\partial \mathcal{B}$. The elements of impulse are eventually transformed into vortex loops of equal impulse. At the same time 'image' vortex loops are created to enforce impermeability at $\partial \mathcal{B}$. In the case of inviscid flow $\mathbf{M}$ is a conserved quantity, and therefore $d \mathbf{M} / d t=0$. In the case of flow where impulse is being created at a solid boundary, we can relate $d \mathbf{M} / d t$ to forces exerted by the fluid on the boundary. 
The total frictional force acting on a stationary bluff body in viscous flow is a sum of two quantities, skin friction and form drag, both of which have direct expression in terms of impulse. Skin friction is expressed as a surface integral

$$
\frac{1}{R e} \int_{\partial \mathcal{B}} \mathbf{n} \times \boldsymbol{\xi} d S
$$

where $\mathbf{n}$ is the normal to the surface. One expects skin friction to be a small component of the total frictional force at higher Reynolds numbers $R e$; at lower $R e$ it shows a pronounced $R e^{-\frac{1}{2}}$ dependence. At higher Re form drag dominates; it is defined as

$$
\int_{\partial \mathcal{B}} p \mathbf{n} d S
$$

where pressure $p=\frac{1}{2}|\mathbf{u}|^{2}+d \phi / d t$. The contribution of the first term $\left(\frac{1}{2}|\mathbf{u}|^{2}\right)$ to drag can be derived from the time derivative of the impulse associated with vorticity in the flow interior, and the contribution of the second term $(d \phi / d t)$ can be derived from the time derivative of the impulse associated with the images of vortex elements [1]. Form drag can be expressed directly in terms of total impulse as

$$
\frac{d \mathbf{M}}{d t}=\frac{1}{2} \frac{d}{d t} \int_{\mathbb{R}^{3}} \mathbf{r} \times \boldsymbol{\xi} d V+\frac{1}{2} \frac{d}{d t} \int_{\mathbb{R}^{3}} \mathbf{r}^{\prime} \times \boldsymbol{\xi}^{\prime} d V
$$

(the primes denoting variables associated with images). Note that if a filament is stretched in an irrotational field, this stretching satisfies

$$
\frac{1}{2} \int_{\mathbb{R}^{3}} \mathbf{r} \times \frac{d \boldsymbol{\xi}}{d t} d V=\frac{1}{2} \int_{\mathbb{R}^{3}} \mathbf{u} \times \boldsymbol{\xi} d V
$$

(see [3] or, for a more elegant demonstration, [8]). Therefore

$$
\frac{d \mathbf{M}}{d t}=\int_{\mathbb{R}^{3}} \mathbf{u} \times \boldsymbol{\xi} d V+\int_{\mathbb{R}^{3}} \mathbf{u}^{\prime} \times \boldsymbol{\xi}^{\prime} d V
$$

In terms of a vorticity field represented by an ensemble of loops of uniform circulation $\Gamma$, this becomes

$$
\frac{d \mathbf{M}}{d t}=\sum_{i} \oint_{i}\left\{\mathbf{u} \times \Gamma d \boldsymbol{\ell}+\mathbf{u}^{\prime} \times \Gamma^{\prime} d \boldsymbol{\ell}^{\prime}\right\}
$$

where summation is over loops.

This can be further approximated for a polygonal representation of the loops by the summation:

$$
\frac{d \mathbf{M}}{d t}=\left\{\sum_{i} \mathbf{u}_{i} \times \Gamma \mathbf{s}_{i}+\mathbf{u}_{i}^{\prime} \times \Gamma^{\prime} \mathbf{s}_{i}^{\prime}\right\}
$$

where $\mathbf{u}_{i}$ is the velocity of the mid-point of the $i$ th segment, and $\mathbf{u}_{i}^{\prime}$ that of the corresponding image segment. Equation 8 is a mid-point quadrature of the line-integration over the loops and its accuracy depends on the refinement of the segments. Higher-order quadrature can also be used. In the context of the present methods based on vortex loops, Eq. 8 offers a numerical advantage, since form drag can be calculated from the Biot-Savart evaluation of $\mathbf{u}$; numerical differentiation in time is avoided. Drag and skin-friction are often expressed as drag coefficients, which, in the case of unit flow over a unit sphere (and for $\rho=1$ ), imply division by half the projected area, $\pi / 2$. 


\section{Choosing $\mathrm{m}$ normal to the wall}

\subsection{Impulse generation}

We can use dimensional considerations the neighbourhood of a solid wall to develop a choice of gauge which will lead us to approximate equations of motion analogous to the boundary layer equations discussed, for example, by Schlichting [13]. Consider a solid boundary at $z=0$ (where $\mathbf{r}=(x, y, z)$ represents the Cartesian position vector). The region $z>0$ is occupied by a fluid with viscosity $\nu$ and a velocity field $\mathbf{u}=\left(u_{x}, u_{y}, u_{z}\right)$. Imagine a thin layer of fluid over the boundary, of thickness $\delta$, such that the following dimensional considerations hold: Lateral length scales are order 1, i.e. $(x, y) \sim O(1)$; the normal length scale is $O(\delta)$. This implies the following: $\partial u_{x} / \partial t \sim O(1)$; $u_{x} \sim O(1) ; \partial u_{x} / \partial x \sim O(1)$; (similarly for $u_{y}$ and $\partial u_{y} / \partial y$ ). The continuity condition implies that $\partial u_{z} / \partial z \sim O(1)$, hence $u_{z} \sim O(\delta)$.

From these considerations we can infer the orders of the components of vorticity $\xi: \xi_{x} \sim O(1 / \delta)$ and $\xi_{y} \sim O(1 / \delta)$ and from the solenoidal property of vorticity we infer $\xi_{z} \sim O(1)$. From the relationship $\boldsymbol{\xi}=\nabla \times \mathbf{m}$ we can infer the order of the components of $\mathbf{m}, m_{x} \sim O(1), m_{y} \sim O(1)$, and $m_{z} \sim O(1 / \delta)$.

Using this dimensional description, we can now examine the three cartesian component equations of (5) and determine their relative orders. Retaining the leading terms of $O(1 / \delta)$ the following scalar transport equation survives,

$$
\frac{D m_{z}}{D t}=-\mathbf{m} \cdot \frac{\partial \mathbf{u}}{\partial z}+\nu \frac{\partial^{2} m_{z}}{\partial z^{2}} .
$$

This equation is reminiscent of the three-dimensional Prandtl equation for a scalar variable, although with an additional term on the right-hand side. Equation 9 has a simple physical interpretation: A vortex loop impinging on a wall is stretched laterally in an amount proportional to the velocity gradient.

Choosing the gauge $\mathbf{m}=m_{z} \hat{\mathbf{z}}$ (i.e. choosing $\mathbf{m}$ normal to the wall) generates the transport equation

$$
\frac{D m_{z}}{D t}=-m_{z} \frac{\partial u_{z}}{\partial z}+\nu \frac{\partial^{2} m_{z}}{\partial z^{2}}
$$

We have just shown that it is self-consistent to choose $\mathbf{m}$ at the wall normal to the wall, provided a boundary layer approximation is used very near to the wall, as must be done anyway to ensure accuracy.

We can find a boundary condition for $m_{z}$ at the wall. Since we have

$$
\boldsymbol{\xi}=\nabla \times m_{z} \hat{\mathbf{z}}
$$

(where $\hat{\mathbf{z}}$ is the unit vector normal to the boundary) we can invert this to determine a condition for $m_{z}$ on the boundary, so that

$$
m_{z}=\int_{o}^{x} \xi_{y} d x-\int_{0}^{y} \xi_{x} d y
$$

where the integration is carried out along the boundary. This expression is similar to that developed in [14] - this is to say the normally-directed vector potential described in [14] can be identified with the normal component of a magnetization vector.

A discussion in reference [7] (pp. 74-75) is relevant also to Eq. 10. We could choose the region in which a numerical boundary layer is confined to be sufficiently thin, specifically $O\left(R e^{-\alpha}\right)$, where $\alpha>\frac{1}{2}$, with $R e$ the Reynolds number. In such a close proximity of the surface the transport is 
predominantly diffusive, and the terms with first-order spatial derivatives in (10) could, as a first approximation, be neglected.

Also, we could approximate wall-vorticity by a first-order difference. Consider in the present cartesian case a boundary layer of thickness $\delta$, with an external tangential velocity field $\left(u_{x}, u_{y}\right)$. We could make the approximation $\boldsymbol{\xi} \simeq \delta^{-1}\left(-u_{y}, u_{x}\right)$. Hence Eq. 11 could be expressed as

$$
m_{z}=\delta^{-1} \int_{0}^{x} u_{x} d x+\delta^{-1} \int_{0}^{y} u_{y} d y
$$

or, for a more general geometry, the normal component of impulse is given by

$$
m_{n}=\delta^{-1} \int_{C} \mathbf{u} \cdot d \mathbf{r}
$$

with $C$ a path taken in the surface. For a closed path in the surface the line integral represents the circulation through the area spanned by the path; due to impermeability, and for sufficiently simple geometries, this closed integral should vanish (for example, if $C$ were in a simply-connected convex surface).

It will be noted that, to Eqs. 11 or 12, attaches an arbitary additive constant of integration which reflects the non-uniqueness of $\mathbf{m}$. The choice of origin for the line-integration (12) is immaterial.

The level curves of $m_{z}$ should have a physical significance. The vorticity, $\boldsymbol{\xi}$, in the boundary layer approximation, is tangential to the boundary. At any point of the boundary $\boldsymbol{\xi}$ must be both normal to $\nabla m_{z}$ and to the normal vector $\hat{\mathbf{z}}$ (recall in the present cartesian case $\boldsymbol{\xi}=\nabla \times m_{z} \hat{\mathbf{z}}=\nabla m_{z} \times \hat{\mathbf{z}}$ ). If we define a unit vector aligned to the local vorticity, i.e. $\hat{\mathbf{q}} \equiv \boldsymbol{\xi} /|\boldsymbol{\xi}|$, then

$$
\hat{\mathbf{q}} \cdot \nabla m_{z}=\frac{\partial m_{z}}{\partial q}=0
$$

This is to say the level curves of $m_{z}$ in the wall are lines of equivorticity.

To summarize, Eq. 12 can be used to evolve magnetization from a no-slip surface. At some time $t$ the no-slip condition fails to be satisfied: The slip field can then be integrated in (12) to produce a value of $m_{n}$ at every point of the surface. Equation 10 represents an equation of motion for impulse density (and this equation may be further simplified to a diffusion equation within an appropriately small distance from the surface).

\subsection{Matching boundary flow to the interior}

We have described a method for determining the distribution of impulse density over a closed solid boundary. A principle of localization can be applied to this distribution in analogy with that used in the construction of vortex sheets to model boundary layer flow. Imagine an impulse density determined at each point of the surface. The surface can be partitioned into tiles, and each tile can be made to bear the appropriate local value of $m_{n}$. For example this value for the $j$ th such tile could be determined from a quadrature version of the line-integration

$$
m_{n}^{(j)}=\sum_{i=1}^{j} \int_{x_{i}}^{x_{i+1}} \xi_{y} d x=\delta^{-1} \sum_{i=1}^{j} \int \mathbf{u}_{i} \cdot d \boldsymbol{\ell}
$$

(taking integration in $x$ to be generic). Each such tile would interact with other tiles according to (10). For small enough values of $\delta$ this is primarily a diffusion normal to the surface. In a random-walk representation of diffusion we would require $\delta$ to be at most several mean-free paths, i.e. some small multiple of $\sqrt{2 d t / R e}$, with $d t$ the time-step. 
During this creation process we can also assign to each tile the circulation required to effect no-slip locally; for a $h \times h$ tile, this is $h\left(-u_{y}, u_{x}\right)$ [5], following the usual vortex sheet methods. The use of a tent-function smoothing kernel leads to a smooth velocity due to sheets at the surface. The back-flow associated with vorticity is reduced, and this leads to greater numerical stability near the wall. Therefore it seems reasonable to use these vortex sheets to determine the velocity induced by tile elements (three-dimensional sheet elements) at the wall.

When tiles diffuse out of the sheet layer, the impulse density attached to each tile is used to determine an interim element of impulse: An 'element' of $\mathbf{M}$ is created by multiplying $m_{n}$ by a volume element $d V=d a \delta$, where $d a$ is the tile area. This element is then transformed into a vortex loop of equal impulse. Thus for a sheet with impulse density $m_{n}$, we construct a vortex loop of circulation $\Gamma$ and spanned area $A_{\text {loop }}$ such that

$$
\Gamma A_{\text {loop }}=m_{n} d V
$$

Equation 13 alone does not determine a vortex loop uniquely. We can create an ensemble of vortex loops of equal circulation, interacting with each other by the Biot-Savart relation. This leads to an N-body calculation which can be accelerated by fast-solver strategies, e.g. the Greengard- Rokhlin multipole fast solver method [10]. Such a strategy has been applied to ensembles of filaments [12].

\subsection{Numerical experiment: flow past a sphere}

To illustrate the algorithm based on $\mathbf{m}$ perpendicular to the wall we consider a uniform flow of unit magnitude into which is placed a unit sphere. Standard vortex blob methods have recently been applied to this problem $[9,15]$. At time $t=0$ we consider the initial flow to be potential. The sphere is partitioned into quadrilateral patches. We perform a series of line-integrations in the surface of the sphere to determine the impulse density there, according to Eq. 12. In spherical coordinates $(r, \theta, \phi)$, and, with a radial impulse density, $m_{r}$, we use trapezoidal quadrature to evaluate

$$
\left.m_{r} \delta\right|_{(\theta, 0)}=\int_{0}^{\theta} \mathbf{u} \cdot d \boldsymbol{\ell}
$$

for $\phi=0$. The slip-field $\mathbf{u}$ is calculated as a combination of potential flow, the velocity induced by previously created sheets, and the field induced by existing filaments and their images. The slip field calculated in this way should satisfy $\mathbf{u} \cdot \mathbf{n}=0$. Subsequently, a series of similar integrations are then performed over $\phi \in(0,2 \pi)$,

$$
\left.m_{r} \delta\right|_{(\theta, \phi)}=\int_{0}^{\phi} \mathbf{u} \cdot d \boldsymbol{\ell}
$$

for a set of values of $\theta \in(0, \pi)$. This generates values of $m_{r} \delta$ at the intersections of the partition mesh. These values can be interpolated to the centroid of each patch. This patch defines the dimensions of a vortex sheet: the tangential velocity at the centroid of this sheet is evaluated; the previously determined impulse density is simply attached to the sheet.

The sheets represent a numerical Prandtl-like boundary layer [5] of thickness $\delta$. They are subject to diffusion normal to the wall. The parameter $\delta$ is chosen sufficiently small so that advective interaction between sheets can be neglected. Upon leaving this layer, each sheet is transformed into a circular vortex loop (more precisely a polygonal approximation to a circle) in a plane tangential to $\partial \mathcal{B}$ and with prescribed circulation $\Gamma$. The radius of this loop is determined by matching the loss of impulse caused by the sheet's departure from the sheet layer; for a given impulse density $m_{r}$ and tile area $d a$, we determine a loop radius $r=\sqrt{\left|m_{r}\right| \delta d a / \Gamma \pi}$. 
Table I: Calculated drag and lift coefficients for steps 9-12.

\begin{tabular}{|c|c|c|c|c|}
\hline Time Step & $\begin{array}{c}\text { No. of } \\
\text { Segments }\end{array}$ & $\begin{array}{c}\text { Lift } \\
(y \text {-compt. })\end{array}$ & $\begin{array}{c}\text { Lift } \\
(z \text {-compt. })\end{array}$ & $\begin{array}{c}\text { Drag } \\
(x \text {-compt. })\end{array}$ \\
\hline 9 & 15,596 & -0.0117 & 0.0282 & 0.2266 \\
\hline 10 & 20,253 & -0.0042 & 0.0185 & 0.1934 \\
\hline 11 & 26,616 & -0.0115 & 0.0239 & 0.1032 \\
\hline 12 & 34,831 & -0.0602 & 0.0513 & 0.0560 \\
\hline
\end{tabular}

In order to preserve impermeability at $\partial \mathcal{B}$, an image loop is created simultaneously inside the sphere. This image is constructed by locating the inversion points of the polygon's vertices. For a segment whose centroid has position vector $\mathbf{r}$, and whose circulation is $\Gamma$, the circulation of the image is $\Gamma^{\prime}=-r \Gamma$ [2]. The velocity field induced by such a collection of segments and images is determined by the usual Biot-Savart algorithm; the Biot-Savart kernel is convolved with a smoothing function. Some attention must be paid to ensuring that smoothing is applied in such a way that the impermeability condition is not violated. The loops are transported in the resulting velocity field (and stretched) using fourth-order Runge-Kutta integrators. As segments are stretched, they are subdivided to maintain their initial level of refinement. The techniques of hairpin removal can also be applied to the ensemble of filaments [6]. Diffusion can be modelled in the flow interior by imparting to each loop a uniform three-dimensional random walk displacement; numerical experiment indicates that the contribution of this to the interior flow is relatively small at high Reynolds number.

One expects such a challenging flow regime to require considerable computational resources, and numerical experiment indeed suggests the algorithm leads quickly to a very large ensemble of loops. The tools of hairpin removal and renormalization can be used to reduce the amount of labor. Figures 1 illustrate the flow evolution over a sphere using the method described in this section. We consider a notional Reynolds number of 200,000 but it should be noted that (in the present method with $\mathbf{m}$ normal to the surface) Reynolds number appears primarily in the thickness of the sheet layer. The uniform incident flow is in the positive $x$-direction. We choose a vortex circulation of $\Gamma=0.1$, a time step $d t=0.2$ which is admittedly large for the present flow, but serves to illustrate the algorithm. Some aspects of Chorin's hairpin removal strategy are invoked (but not reconnection and deconnection). The sphere is partitioned using an icosahedron recursively refined three times, this is to say into 1280 surface patches. At the conclusion of step 9 there are 3059 sheets in the sheet layer; after step 12 there are 4070 sheets. The number of filaments in the flow interior for the time-steps shown in Fig. 1 are presented in Table I, together with drag and lift coefficients calculated using the formulae of Section 4 . At step 12 the $x$-component of skin-friction calculated from (7) is $0.07 \%$ of total drag.

The experimental value of drag coefficient expected for such a flow is about 0.5. Of course the present calculation would represent at best an instantaneous measurement of drag in an initial flow, where the wake is not fully developed. A more considered calculation of drag must await a solution of the problem over longer time, which is in progress. One can note in Fig. 1 the formation of a shedding pentagonal vortex ring structure. These computations were performed on a Cray- 2 . 


\section{Choosing $\mathrm{m}$ parallel to the wall}

We have described in Section 5 a method in which the concept of fluid impulse of compact support is exploited to match a partitioned vortex sheet which has been created at the wall to closed vortex loops in the flow interior. An alternative approach to modelling the effect of a solid boundary on flow is to model the wall forces directly as "impulse creation". A strategy to express the physics associated with this process can be stated as follows. At time $t$ at some point on the boundary there exists a tangential slip velocity $\mathbf{u}_{\text {slip }}$. An element of impulse density can be created at this point such that $\mathbf{m}=-\mathbf{u}_{\text {slip }}$, thus establishing no-slip there at that instant. This corresponds to an alternative gauge in which $\mathbf{m}$ is parallel to the wall.

This element of impulse density is subject to evolution according to (5). In the immediate neighbourhood of the wall this can be approximated as a diffusion normal to the surface. In particular, the element can diffuse through a viscous sheet layer of thickness $\delta$, and the fluid impulse, $\mathbf{M}$, associated with an element of surface area $d a$, leaving this layer is $\mathbf{M}=\mathbf{m} d a \delta$. Upon entering the interior this element can be equated to a vortex loop of equivalent impulse; for example, this could be a circular loop of radius

$$
r=\sqrt{\frac{|\mathbf{m}| d a \delta}{\Gamma \pi}}
$$

The plane of this loop will be oriented vertically to the wall, and normal to the direction of slip flow at the point of creation. (Note that this is in contrast to the method described in Section 5.) One way of implementing this idea is to first create vortex tiles, calculate the impulse associated with them (which is, as time progresses, the created impulse density times the volume traversed between tile and wall), and then, at the edge of the numerical boundary layer, we can replace the tile by an equivalent element of impulse in the form of a vortex loop.

An impulse-creation strategy such as this has obvious attractions: the creation principle is "localized", and this is more consistent with the idea of localized Lagrangian elements. No lineintegration (and thus no corresponding numerical quadrature) is required in the boundary - such as that of Eq. 12 - to determine the closure of closed loop filaments. The creation process is a statement of momentum conservation. On the other hand, the vorticity created using $\mathbf{m}$ parallel to the boundary admits a more complex structure than that associated with $\mathbf{m}$ normal to the boundary. The possibility of normal vorticity at the wall itself is automatically included. Furthermore, the scale of the viscous sheet layer (itself typically a function of Reynolds number) enters into the scale of the created vortex loops. (Again this is in contrast with the method of Section 5.)

\section{In conclusion}

The difference between the two methods presented here is a difference between two choices of gauge. A partition of vorticity into vortex loops is not unique (vortex loops can be connected and disconnected in such a way that the vorticity field is left undisturbed), and to each set of vortex loops there corresponds a set of spanning surfaces and therefore a set of magnetization fields. All these fields differ from each other by a choice of gauge.

The problem of picking the gauge that is most useful numerically remains to be solved; presumably numerical experiment will shed light on this question, and initial experiments are in progress. It is likely that the answer is problem-dependent. Whatever the gauge, the use of vortex loops and impulse considerations near walls allows one to create vorticity in a manner that is divergence-free, which simplifies the application of hairpin removal and renormalization algorithms, and which is likely to lead to simpler and more accurate three-dimensional particle methods. 


\section{Acknowledgements}

Brian Davila of the L.B.N.L. Computer Graphics Group is thanked for his assistance. The authors also thank G. Pringle and D. McKie for their help with various tasks.

\section{References}

[1] Bickley, W.G., "The Influence of Vortices upon the Resistance Experienced by Solids Moving through a Liquid," Proc. Roy. Soc. Lond. A, 119, pp. 146-156, 1928.

[2] Butler, S., "A Note on Stokes' Stream Function for Motion with a Spherical Boundary," Proc. Cam. Phil. Soc., 49, pp. 169-174, 1952.

[3] Buttke, T.F., "Lagrangian Numerical Methods which Preserve the Hamiltonian Structure of Incompressible Fluid Flow," NASA ASI Series, 395, edited by J.T. Beale et al., pp. 301-306, 1993.

[4] Buttke, T.F. and Chorin, A.J., "Turbulence Calculations in Magnetization Variables," Appl. Num. Meth., 112, pp. 47-54, 1993.

[5] Chorin, A.J., "Vortex Models and Boundary Layer Instability," SIAM J. Sci. Stat. Comp., 1, pp. 1-21, 1980.

[6] Chorin, A.J., "Hairpin Removal in Vortex Interactions II," J. Comp. Phys., 107, pp. 1-9, 1993.

[7] Chorin, A.J. and Marsden, J.E., A Mathematical Introduction to Fluid Mechanics, SpringerVerlag, N.Y., third edition, 1993.

[8] Cortez, R., Impulse-Based Methods for Fluid Flow, Ph.D. thesis, University of California, Berkeley, Mathematics Department, 1995.

[9] Fishelov, D., "Simulation of Three-Dimensional Turbulent Flow in Non-cartesian Geometry," J. Comp. Phys., 115, pp. 249-266, 1994.

[10] Greengard, L. and Rokhlin, V., "A Fast Algorithm for Particle Simulations," J. Comp. Phys., 73, pp. 325-348, 1987.

[11] Jackson, J.D., Classical Electrodynamics, John Wiley, N.Y., 1975.

[12] Pringle, G.J., Study of Three-Dimensional Flow using Fast Parallel Particle Algorithms, Ph.D. thesis, Napier University, Edinburg, 1994.

[13] Schlichting, H., Boundary Layer Theory, McGraw Hill, N.Y., 1968.

[14] Summers, D., An Algorithm for Vortex Loop Generation, Technical Report 31367, L.B.L., Berkeley CA, 1991.

[15] Winckelmans, G.S., Salmon, J.K., Warren, M.S. and Leonard, A., "The Fast Solution of ThreeDimensional Fluid Dynamical n-body Problems using Parallel Tree Codes: Vortex Element Method and Boundary Element Method," Proc. 7th SIAM Conf. Parallel Processing for Scientific Computing, edited by Bailey et al., pp. 301-306, 1994. 

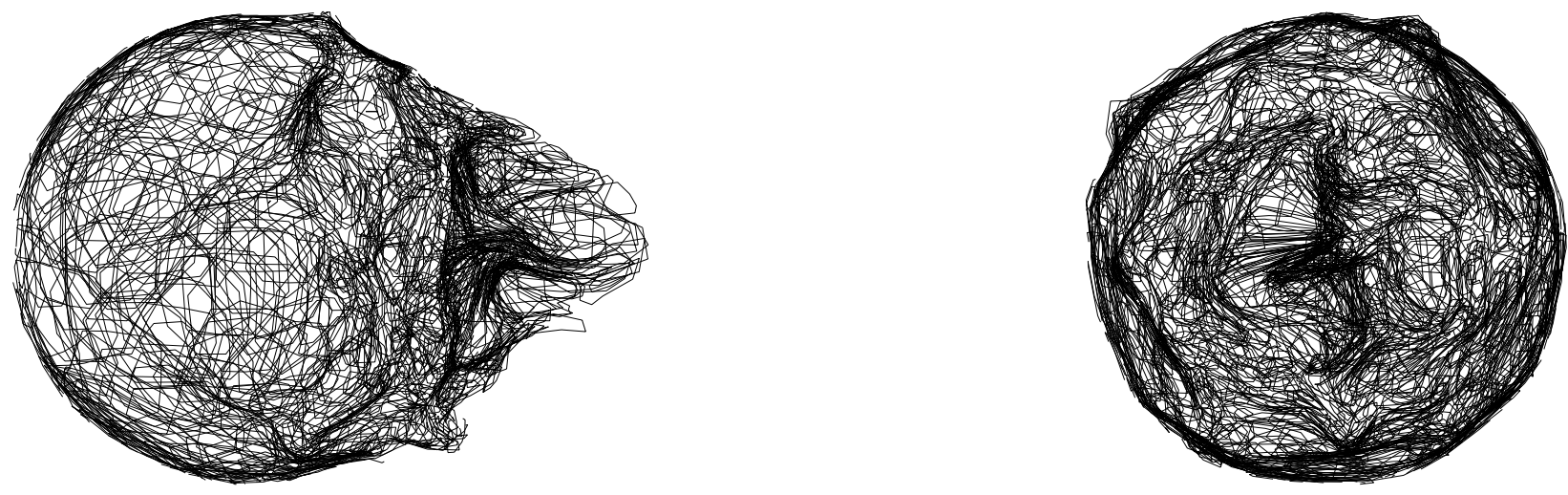

Step 9 (side profile)

Step 9 (lee view)
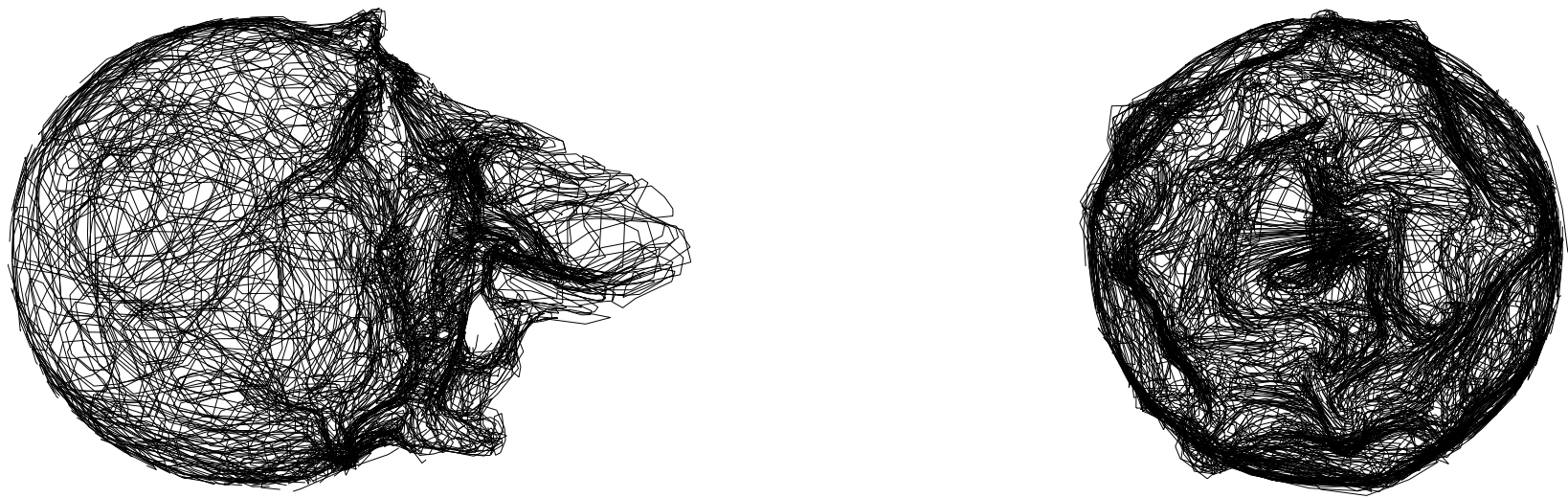

Step 10 (side profile)

Step 10 (lee view)

Figure 1: (a) Flow over a sphere: vortex filament evolution for time steps 9 and 10 with impulse density $\mathbf{m}$ normal to the surface. Time increment $d t=0.2$, circulation $\Gamma=0.1$, cut-off $\delta=0.2$, surface partition is 1280 patches. 

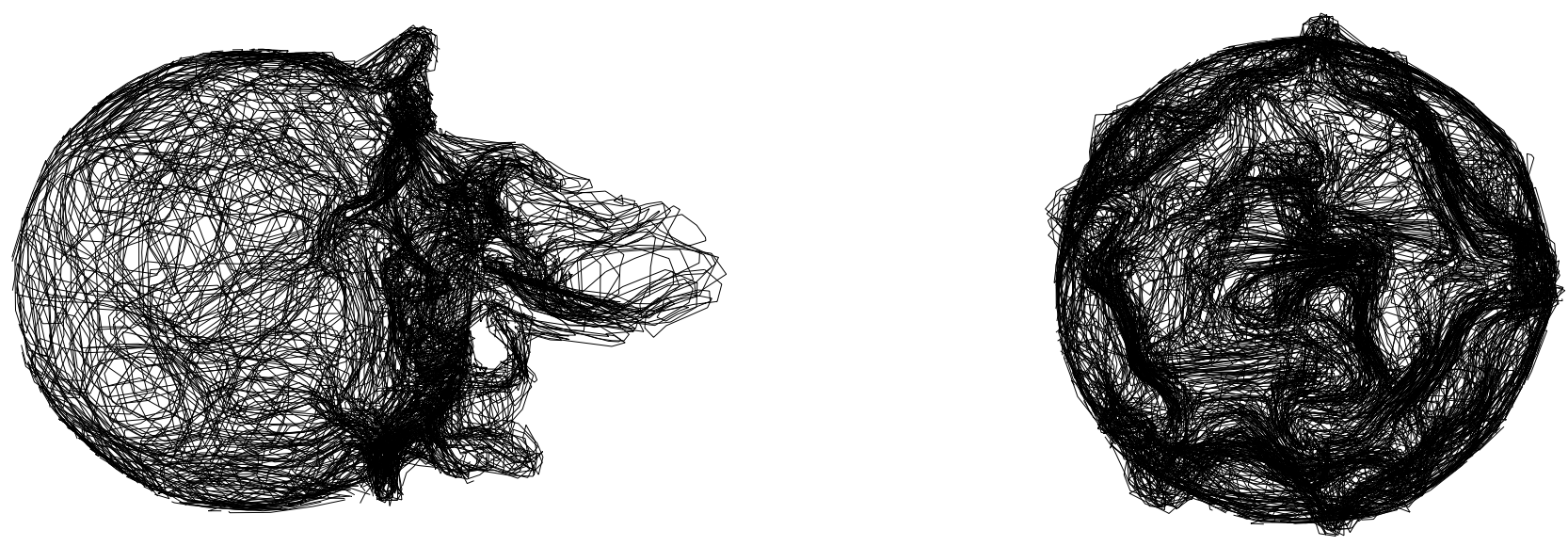

Step 11 (side profile)

Step 11 (lee view)
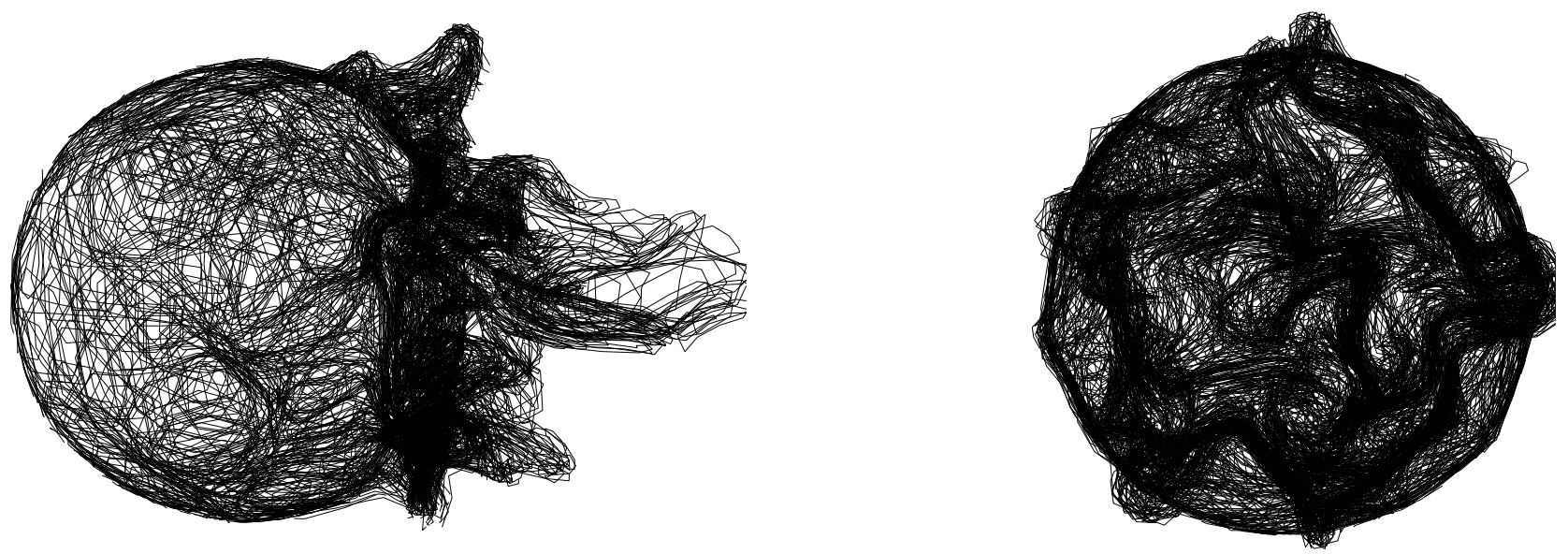

Figure 1: (b) Steps 11 and 12 continuing from Fig. 1a. 